\title{
Relation of coronary flow pattern to myocardial blush grade in patients with first acute myocardial infarction
}

\author{
R Hoffmann, P Haager, W Lepper, A Franke, P Hanrath
}

See end of article for authors' affiliations

\section{Correspondence to:}

Dr Rainer Hoffmann,

Medical Clinic I, University

RWTH Aachen,

Pauwelsstrasse 30, 52057

Aachen, Germany;

rhoffmann@ukaachen.de

Accepted 30 March 2003
Background: Analysis of myocardial blush grade (MBG) and coronary flow velocity pattern has been used to obtain direct or indirect information about microvascular damage and reperfusion injury after percutaneous transluminal coronary angiography for acute myocardial infarction.

Objective: To evaluate the relation between coronary blood flow velocity pattern and MBG immediately after angioplasty plus stenting for acute myocardial infarction.

Design: The coronary blood flow velocity pattern in the infarct related artery was determined immediately after angioplasty in 35 patients with their first acute myocardial infarct using a Doppler guide wire. Measurements were related to MBG as a direct index of microvascular function in the infarct zone.

Results: Coronary flow velocity patterns were different between patients with absent myocardial blush $(n=14)$, reduced blush $(n=7)$, or normal blush $(n=14)$. The following variables (mean (SD)) differed significantly between the three groups: systolic peak flow velocity $(\mathrm{cm} / \mathrm{s})$ : absent blush 10.9 (4.2), reduced blush 14.2 (6.4), normal blush 19.2 (11.2); $p=0.036$; diastolic deceleration rate (ms): absent blush 103 (58), reduced blush 80 (65), normal blush 50 (19); $p=0.025$; and diastolic-systolic velocity ratio: absent blush 4.06 (2.18), reduced blush 2.02 (0.55), normal blush 1.88 (1.03); $p=0.002$. In a multivariate analysis $M B G$ was the only variable with a significant impact on the diastolic deceleration rate $(p=0.034$, while age, infarct location, time to revascularisation, infarct vessel diameter, and maximum creatine kinase had no significant impact.

Conclusions: The coronary flow velocity pattern in the infarct related epicardial artery is primarily determined by the microvascular function of the dependent myocardium, as reflected by MBG.
I $\mathrm{n}$ addition to patency of the infarct related artery, adequate microcirculation at myocardial tissue level has become a major objective in the treatment of acute myocardial infarction. ${ }^{1-3}$ Multiple indices have been suggested to evaluate microvascular function. There are some that allow direct assessment of microvascular function in the myocardium subtended by the infarct related artery, such as contrast echocardiography and myocardial blush grade (MBG). ${ }^{4-8}$ Other variables such as TIMI (thrombolysis in myocardial infarction) frame count, coronary flow reserve, and analysis of coronary blood flow patterns only indirectly reflect microvascular function by revealing the blood flow characteristics in the coronary artery supplying the affected myocardium. ${ }^{9-11}$ The coronary blood flow pattern in the infarct related artery is of considerable interest in assessing reperfusion and predicting left ventricular function at follow up. The acute coronary blood flow pattern of patients with revascularised but nonreperfused acute myocardial infarction is characterised by the appearance of early systolic retrograde flow, diminished systolic antegrade flow, and rapid deceleration of diastolic flow. ${ }^{11-13}$ These findings are thought to be the result of altered impedance and blood volume capacity in the subtended myocardium. However, the relation between microvascular dysfunction at tissue level and the resulting alterations in blood flow characteristics has not been studied in detail.

In the study reported here, on patients who had received angioplasty with stenting for acute myocardial infarction, we evaluated the association between depressed microvascular function at myocardial tissue level, as determined by MBG, and alterations in coronary flow pattern in the epicardial artery supplying the damaged myocardium.

\section{METHODS}

\section{Study population}

We studied 35 consecutive patients with their first acute myocardial infarct undergoing revascularisation by primary angioplasty within six hours of the onset of pain. Inclusion criteria were typical anginal pain lasting more than 30 minutes and ST segment elevation of more than $0.2 \mathrm{mV}$ in at least two contiguous ECG leads. The research protocol was approved by the local institutional ethics committees.

\section{Coronary angioplasty and coronary blood flow analysis} Before catheterisation, all patients received $10000 \mathrm{U}$ of heparin and $500 \mathrm{mg}$ aspirin intravenously. Either ticlopidine $500 \mathrm{mg}$ or clopidogrel $300 \mathrm{mg}$ were given orally as a loading dose before the intervention. Before or during the coronary intervention, weight adjusted abciximab was given at the discretion of the operator. Successful stent placement was undertaken in all patients. Flow in the infarct vessel after angioplasty was graded using the TIMI flow classification. ${ }^{14}$ Off-line quantitative coronary angiographic analysis was done using an automated edge detection system (CAAS II System, PieMedical, Maastricht, Netherlands). Variables determined were reference diameter, minimum lumen diameter, and diameter stenosis postintervention.

Within five minutes of completion of interventional recanalisation, the guide wire was exchanged for a 0.014 inch intracoronary Doppler tipped flow wire (12 MHz, FloWire, Cardiometrics Inc, Mountain View, California, USA) to make intracoronary flow measurements. Placing the tip just proximal to the site of recanalisation ensured that the sampling volume corresponded to the site of occlusion. This allowed assessment of flow to the entire region at risk. ECG and blood pressure were monitored continuously. Doppler flow velocity spectra were analysed off-line to determine the

Abbreviations: GWMI, global wall motion index; MBG, myocardial blush grade; TIMI, thrombolysis in myocardial infarction 
Table 1 Clinical and angiographic data and functional outcome of patients belonging to the three reperfusion groups defined by myocardial blush grade

\begin{tabular}{lllll}
\hline & MBG 0/1 $(\mathrm{n}=14)$ & MBG 2 $(\mathrm{n}=7)$ & MBG $3(\mathrm{n}=14)$ & $\mathrm{p}$ Value \\
\hline Age (years) & $66(10)$ & $63(9)$ & $61(12)$ & 0.398 \\
Male & $10 / 14$ & $4 / 7$ & $7 / 14$ & 0.508 \\
Time to reperfusion (hours) & $3.87(1.23)$ & $4.97(1.43)$ & $4.54(1.37)$ & 0.126 \\
Infarct location: anterior/inferior & $9 / 5$ & $2 / 5$ & $4 / 10$ & 0.124 \\
Peak creatine kinase (U/I) & $1867(1057)$ & $1638(798)$ & $1064(798)$ & 0.075 \\
TIMl 3 after PTCA & $8 / 14$ & $6 / 7$ & $12 / 14$ & 0.176 \\
Number of diseased vessels & $2.32(0.87)$ & $1.98(0.65)$ & $2.12(0.74)$ & 0.322 \\
Reference diameter (mm) & $3.4(0.7)$ & $3.1(0.4)$ & $3.0(0.6)$ & 0.224 \\
Diameter stenosis post PTCA (\%) & $8.6(6.5)$ & $11.6(4.5)$ & $9.2(5.7)$ & 0.263 \\
Abciximab & $7 / 14$ & $2 / 7$ & $3 / 14$ & 0.272 \\
RWMI before PTCA & $2.78(0.18)$ & $2.82(0.17)$ & $2.63(0.51)$ & 0.389 \\
RWMl after 4 weeks & $2.64(0.33)$ & $1.82(0.57)$ & $2.07(0.49)$ & 0.001 \\
GWMI before PTCA & $1.93(0.25)$ & $1.69(0.16)$ & $1.62(0.21)$ & 0.002 \\
GWMI after 4 weeks & $1.90(0.31)$ & $1.34(0.25)$ & $1.44(0.23)$ & $<0.001$ \\
\hline
\end{tabular}

Values are mean (SD) or $\mathrm{n}$.

GWMI, global wall motion score index; MBG, myocardial blush grade; PTCA, percutaneous transluminal coronary angioplasty; RWMI, regional wall motion score index; TIMI, thrombolysis in myocardial infarction study grade.

following indices: peak and mean antegrade coronary flow velocity in systole and diastole, systolic and diastolic flow duration, the calculated ratio of mean diastolic to systolic flow velocity, and the deceleration time of diastolic velocity. The rate of decline of flow velocity in diastole was calculated as the diastolic deceleration rate. A pathologically rapid retrograde flow in early systole was defined as a negative peak velocity of $\geqslant 10 \mathrm{~cm} / \mathrm{s}$ and a duration of $\geqslant 60 \mathrm{~ms}$, as described previously. ${ }^{11}$ All measurements were repeated three times and the mean value was used.

\section{Myocardial blush grade analysis}

MBG was determined off-line by a blinded experienced interventionalist on the final angiogram, from a visual assessment of contrast opacification of the myocardial territory subtended by the infarct vessel, as described previously. ${ }^{4}$ MBG was defined as follows: 0 , no myocardial blush or contrast density; 1, minimal myocardial blush or contrast density; 2, moderate myocardial blush or contrast density but less than that obtained during angiography of a contralateral or ipsilateral non-infarct related coronary artery; 3 , normal myocardial blush or contrast density, comparable with that obtained during angiography of a contralateral or ipsilateral non-infarctrelated coronary artery. MBG 0 and 1 were combined in the results. From multiple orthogonal projections, the single view was chosen that best isolated the myocardial infarct zone in question. Intraobserver and interobserver variability in the assessment of MBG were determined from a random sample of 15 films scored by $\mathrm{RH}$ and $\mathrm{PhH}$. Assessment of intraobserver variability produced a $\kappa$ value of 0.91 , and assessment of interobserver variability, a $\kappa$ value of 0.88 .

\section{Left ventricular function at follow up}

Left ventricular wall motion at four weeks was analysed according to the 16 segment model of the American Society of Echocardiography, and global and regional wall motion indices were calculated. ${ }^{15}$ According to their left ventricular function at four weeks, patients were defined as having preserved left ventricular function (global wall motion index $($ GWMI $)<1.5)$ or depressed function $($ GWMI $\geqslant 1.5) .{ }^{16}$

\section{Statistical analysis}

Continuous variables are presented as mean (SD) and were compared using Student's $t$ test or the Wilcoxon test as appropriate. Dichotomous variables were compared using $\chi^{2}$ statistics or Fisher's exact test. Pearson and Spearman correlation coefficients were calculated as appropriate. Univariate and multivariate regression analysis was used to identify predictors of coronary flow velocity pattern. The diastolic deceleration rate was used as the variable reflecting the coronary flow velocity pattern. Variables included in the model were myocardial blush grade, age, sex, reference vessel diameter, infarct location, and time to revascularisation. Probability values of $\mathrm{p} \leqslant 0.05$ were regarded as significant. All statistical analysis was done using SAS V8.2 software (SAS Institute Inc, Cary, North Carolina, USA).

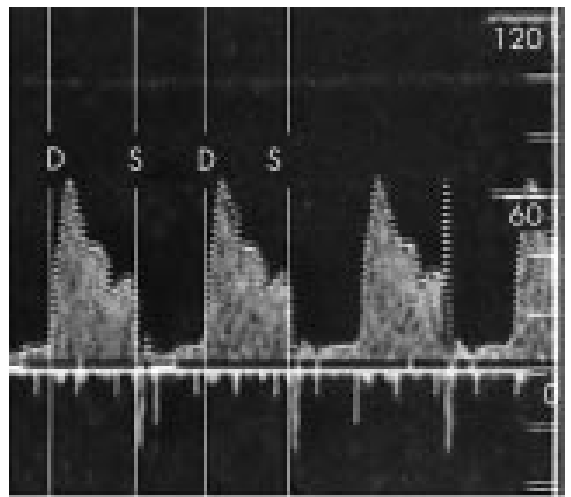

MGB 1

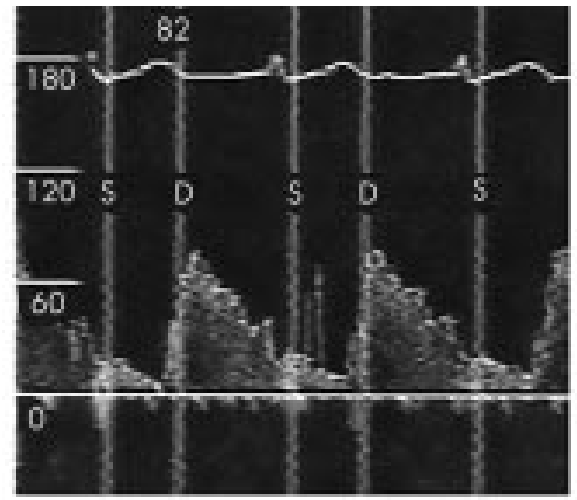

NGB 2

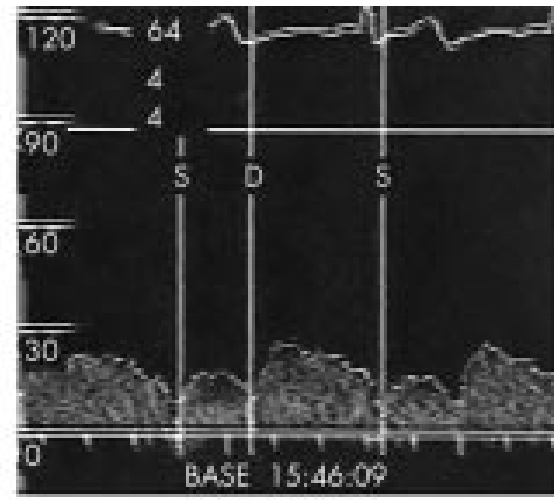

MGB 3

Figure 1 Coronary flow velocity pattern of a patient with severely abnormal myocardial perfusion (left panel) defined by a myocardial blush grade (MBG) of 1; of a patient with impaired myocardial perfusion (middle panel) defined by an MBG of 2; and of a patient with normal perfusion (right panel) defined by an MBG of 3 . 
Table 2 Coronary flow velocity data and haemodynamics immediately after intervention in patients in relation to the three reperfusion groups, as defined by the angiographic blush of the myocardium subtended by the infarct related artery

\begin{tabular}{|c|c|c|c|c|}
\hline & MBG $0 / 1 \quad(n=14)$ & MBG $2(n=7)$ & MBG $3(n=14)$ & $\mathrm{p}$ Value \\
\hline Heart rate (beats/min) & $82(9)$ & $79(16)$ & $80(13)$ & 0.882 \\
\hline Systolic blood pressure $(\mathrm{mm} \mathrm{Hg})$ & $130(27)$ & $114(24)$ & $121(20)$ & 0.135 \\
\hline Diastolic blood pressure $(\mathrm{mm} \mathrm{Hg})$ & $82(16)$ & $72(12)$ & $74(10)$ & 0.218 \\
\hline \multicolumn{5}{|l|}{ Systolic coronary flow } \\
\hline $\mathrm{PV}(\mathrm{cm} / \mathrm{s})$ & $10.9(4.2)$ & $14.2(6.4)$ & $19.2(11.2)$ & 0.036 \\
\hline $\mathrm{FD}(\mathrm{ms})$ & $198(99)$ & $360(109)$ & $289(25)$ & $<0.001$ \\
\hline Number of ESFR & $8 / 14$ & $0 / 7$ & $0 / 14$ & 0.043 \\
\hline \multicolumn{5}{|l|}{ Diastolic coronary flow } \\
\hline $\mathrm{PV}(\mathrm{cm} / \mathrm{s})$ & $37.3(18.6)$ & $31.4(18.2)$ & $33.6(13.1)$ & 0.701 \\
\hline $\mathrm{FD}(\mathrm{ms})$ & $431(65)$ & $494(158)$ & $468(64)$ & 0.297 \\
\hline $\operatorname{DDR}\left(\mathrm{cm} / \mathrm{s}^{2}\right)$ & $103(58)$ & $80(65)$ & $50(19)$ & 0.025 \\
\hline DDT (ms) & $478(271)$ & $671(329)$ & $713(249)$ & 0.076 \\
\hline DSVR & $4.06(2.18)$ & $2.02(0.55)$ & $1.88(1.03)$ & 0.002 \\
\hline \multicolumn{5}{|c|}{$\begin{array}{l}\text { Values are mean }(S D) \text { or } n \text {. } \\
\text { DDR, diastolic deceleration rate; DDT, diastolic deceleration time; DSVR, diastolic-systolic velocity ratio; FD, } \\
\text { flow duration; MBG, myocardial blush grade; Number of ESFR, number of patients with early systolic } \\
\text { retrograde flow; PV, peak flow velocity. }\end{array}$} \\
\hline
\end{tabular}

\section{RESULTS}

\section{Study cohort, procedural outcome}

Depending on the MBG examination 14 patients were assigned to a low reperfusion group, defined by an MBG of 0 or 1 , seven were assigned to an intermediate reperfusion group, defined by an MBG of 2, and 14 were assigned to a preserved perfusion group, defined by an MBG of 3. Baseline clinical, angiographic, and functional outcome data are shown in table 1 .

At a four week follow up assessment, regional and global wall motion indices were impaired and peak creatine kinase tended to be higher in patients with a lower reperfusion level, as defined by the MBG (table 1).

\section{Coronary flow velocity patterns related to myocardial blush grade}

The three myocardial reperfusion groups defined by the MBG did not differ with respect to arterial blood pressure or heart rate during the intracoronary flow velocity measurements (table 1). There were also no differences with regard to the administration of abciximab. Figure 1 shows typical coronary blood flow velocity patterns in patients from the three reperfusion groups as defined by MBG.
Table 2 shows data on the different coronary flow velocity pattern indices related to the reperfusion level, as determined by angiographic MBG. Patients with a low reperfusion level defined by the MBG had more frequent early systolic flow reversal, a shorter systolic flow duration, a lower average systolic peak velocity, a higher diastolic deceleration rate, a higher diastolic-systolic velocity ratio, and a tendency for a shorter diastolic deceleration time compared with patients with better reperfusion levels (fig 2).

\section{Coronary flow velocity pattern related to left ventricular function at follow up}

Average GWMI decreased from $1.76(0.26)$ after revascularisation to $1.60(0.36)$ at the four weeks follow up $(\mathrm{p}<0.001)$. Twenty two patients had depressed left ventricular function (GWMI $\geqslant 1.5$ ) at four weeks, and 13 had preserved left ventricular function $($ GWMI $<1.5)$. Compared with patients with a GWMI of $<1.5$, patients with a GWMI of $\geqslant 1.5$ at the four week follow up had a lower peak systolic velocity (11.6 (4.9) $v 20.4(10.9) \mathrm{cm} / \mathrm{s}, \mathrm{p}=0.002)$, a lower systolic flow duration $(242(98) \vee 311(92) \mathrm{ms}, \mathrm{p}=0.046)$, a higher diastolic deceleration rate $\left(93.9 \quad(58.2) \quad v \quad 48.9 \quad(22.4) \mathrm{cm} / \mathrm{s}^{2}\right.$, $\mathrm{p}=0.012)$, a shorter diastolic deceleration time $(523(313) v$
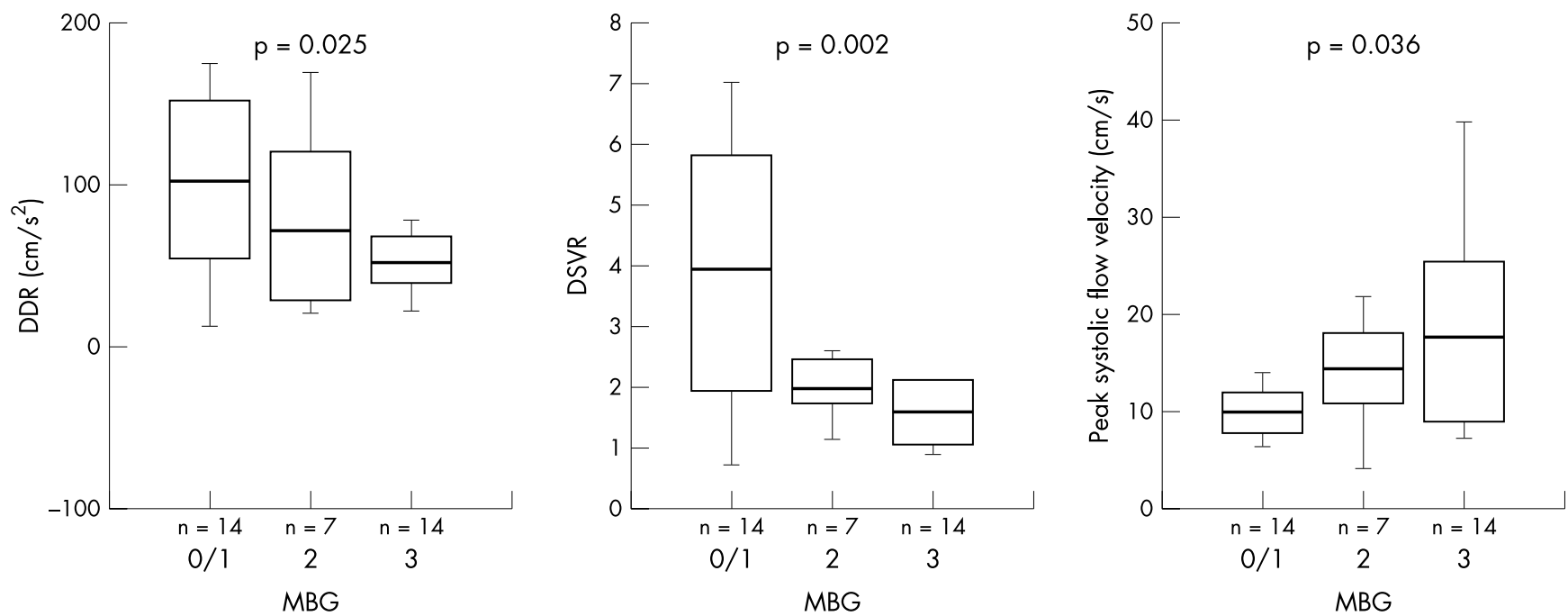

Figure 2 Box plots of diastolic deceleration rate (DDR), diastolic-systolic velocity ratio (DSVR), and peak systolic flow velocity for the three different reperfusion groups, as defined by the angiographic blush grade of the myocardium subtended to the infarct related artery. There are significant differences in the coronary flow velocity pattern indices between the three perfusion levels defined by the myocardial blush grade. 
760 (161) ms, p = 0.017), and a greater diastolic-systolic velocity ratio $(3.42(2.03) v 1.69(0.59), \mathrm{p}=0.005)$.

\section{Variables affecting flow velocity pattern}

Multivariate analysis showed that MBG was the only variable that was correlated with diastolic deceleration rate $(p=0.036)$ as an important index of the coronary flow velocity pattern, while age, sex, infarct location, time to revascularisation, reference vessel diameter, and peak serum creatine kinase had no significant impact on the diastolic deceleration rate.

\section{DISCUSSION}

Our study showed first, an association between several indices of the coronary flow velocity pattern and left ventricular function at follow up; second, significant differences in the coronary blood flow pattern between different reperfusion levels after acute myocardial infarction defined by MBG; and third, that reperfusion at myocardial tissue level, as assessed by $\mathrm{MBG}$, was the strongest predictor of the diastolic deceleration rate as an index of the coronary blood flow pattern in the infarct related artery.

\section{Microvascular dysfunction after acute myocardial infarction}

A possible lack of reperfusion at myocardial tissue level, despite a patent infarct related vessel with TIMI grade 3 flow after revascularisation for acute myocardial infarction, was first described by Ito and colleagues using myocardial contrast echocardiography. ${ }^{1}$ This "no reflow" phenomenon is thought to be the result of microvascular dysfunction. It constitutes a marker of more extensive myocardial tissue damage and is associated with poor functional recovery and worse outcome after acute myocardial infarction. ${ }^{245}{ }^{17}$ Several indices have been used to describe a possible lack of reperfusion at myocardial tissue level. Among these are the MBG, the coronary flow reserve, and alterations in the coronary flow velocity pattern. ${ }^{4-6-11}$ Myocardial blush, the angiographic evidence of myocardial perfusion in the myocardial bed subtended by the infarct artery, was described by van't Hof and colleagues. ${ }^{4}$ The myocardial blush scale has been found to relate to the enzymatic infarct size in patients after primary angioplasty for acute myocardial infarction, ${ }^{10}{ }^{11}$ and to have a better predictive value for long term mortality than Killip class, TIMI grade flow, left ventricular ejection fraction, or other clinical variables. The value of this index for classifying the level of reperfusion after acute myocardial infarction has been demonstrated in several studies. ${ }^{5618}$

\section{Coronary flow pattern after myocardial infarction}

Iwakura and colleagues were the first to report alterations in coronary flow pattern in patients with persistent perfusion defects after acute myocardial infarction. ${ }^{11}$ They showed impaired systolic antegrade flow, the appearance of abnormal retrograde flow in early systole, and rapid deceleration of the diastolic flow velocity in patients with persistent perfusion defects defined by myocardial contrast echocardiography. An increase in microvascular impedance and a decrease in intramyocardial blood pool volume are thought to be sequels of myocardial tissue damage. The increased microvascular impedance has been used to explain the impaired systolic antegrade flow as well as the systolic retrograde flow. Obstruction of the microvasculature with subsequent high impedance results in the inability to squeeze blood forward into the venous circulation during systole. As a result it is pushed back to the epicardial coronary artery to produce early systolic retrograde flow. The reduced intramyocardial blood pool which fills rapidly during diastole has been used to explain the rapid decline of diastolic velocity. ${ }^{11}$ Subsequent analysis has shown that early systolic retrograde flow, a low systolic peak velocity, and a low diastolic deceleration time are predictors of functional recovery after acute myocardial infarction. ${ }^{12}{ }^{19-21}$ Our present study confirms a significant difference in coronary flow velocity pattern between patients with preserved and depressed left ventricular function at a four week follow up after acute myocardial infarction. Differences in flow velocity pattern affected several variables including the peak systolic flow, systolic flow duration, diastolic deceleration rate, and diastolic deceleration time, as well as the diastolic-systolic flow ratio.

\section{Predictors of coronary flow velocity pattern}

While previous studies have shown that the coronary flow pattern is a predictor of left ventricular function at follow up, and differs between patients with and without reperfusion as defined by myocardial contrast echocardiography, the interaction between MBG - as an index of reperfusion level—and the coronary flow velocity pattern has not been defined. The mechanisms that are probably responsible for altered coronary flow velocity patterns have been described. ${ }^{11}$ However, the importance of reperfusion status at myocardial tissue level in comparison with other indices used to define acute myocardial infarction has not been evaluated in detail. Coronary flow velocity pattern and MBG reflect different aspects of impaired microvascular function after acute myocardial infarction. Myocardial blush analysis gives direct evidence of microvascular perfusion at myocardial tissue level. Microvascular disruption, plugging caused by thromboembolic debris, and endothelial and myocardial oedema may reduce the blood volume and microvascular blood flow, resulting in a low degree of myocardial blush. In contrast, the coronary flow velocity pattern in the epicardial coronary artery supplying the infarcted myocardium is an index that only indirectly reflects the status of the microcirculation. Our study shows the strong relation between the pattern of coronary flow and microvascular function downstream to the epicardial artery. Stepwise grading of microvascular integrity using MBG was reflected by a stepwise alteration in coronary flow velocity pattern. Furthermore, in a multivariate analysis the myocardial tissue perfusion level defined by the MBG was found to have a greater impact on the flow pattern than other variables used to describe a myocardial infarct, such as infarct location, time to reperfusion, maximum creatine kinase level, and the size of the infarct related artery.

\section{Limitations}

The number of patients included in this study-although larger than in previous studies analysing the coronary flow velocity pattern-was still limited. The resulting lack of statistical power might have prevented covariates other than MBG from having an impact on the coronary flow pattern. This limitation does not undermine the finding that the blush grade is obviously the strongest predictor of the coronary flow pattern.

The coronary blood flow velocity pattern differs between the different coronary arteries. In the right coronary artery systolic blood flow is relatively augmented. It is therefore possible that the differences in systolic peak flow velocity and diastolic-systolic velocity ratio between the three reperfusion groups might in part be attributed to the different infarct locations in the three groups. However, this limitation does not affect other coronary flow pattern indices.

We used the myocardial blush grade classification described by van't Hof and colleagues. ${ }^{4}$ A slightly different classification has been described by Gibson and associates. ${ }^{5}$ It is possible that the relation between coronary flow pattern and myocardial blush would have been slightly different if the classification described by Gibson and associates had been used. However, the classification by van't Hof and colleagues has been adopted in other recent studies. ${ }^{6} 18$ 


\section{Conclusions}

The coronary flow velocity pattern in the infarct related epicardial artery is primarily determined by the microvascular function of the dependent myocardium reflected by the MBG.

\section{Authors' affiliations}

R Hoffmann, P Haager, W Lepper, A Franke, P Hanrath, Medical Clinic I, University Hospital RWTH Aachen, Germany

\section{REFERENCES}

1 Ito $H$, Tomooka T, Sakai N, et al. Lack of myocardial perfusion immediately after successful thrombolysis. A predictor of poor recovery of left ventricular function in anterior myocardial infarction. Circulation 1992:85:1699-705.

2 Roe MT, Ohman EM, Maas ACP, et al. Shifting the open-artery hypothesis downstream: the quest for optimal reperfusion. J Am Coll Cardiol 2001;37:9-18.

3 Schomig A, Kastrati A, Dirschinger J, et al. Coronary stenting plus platelet glycoprotein Ilb/Illa blockade compared with tissue plasminogen activator in acute myocardial infarction. Stent versus thrombolysis for occluded coronary arteries in patients with acute myocardial infarction study investigators. N Engl J Med 2000;343:1815.

4 van't Hof AW, Liem A, Suryapranata $H$, et al. Angiographic assessment of myocardial reperfusion in patients treated with primary angioplasty for acute myocardial infarction: myocardial blush grade. Zwolle myocardial infarction study group. Circulation 1998;97:2302-6.

5 Gibson CM, Cannon CP, Murphy SA, et al. Relationship of TIMI myocardial perfusion grade to mortality after administration of thrombolytic drugs. Circulation 2000;101:125-30.

6 Stone GW, Peterson MA, Lansky AJ, et al. Impact of normalized myocardial perfusion after successful angioplasty in acute myocardial infarction. J Am Coll Cardiol 2002;39:591-7.

7 Firschke C, Lindner JR, Wei K, et al. Myocardial perfusion imaging in the setting of coronary artery stenosis and acute myocardial infarction using venous injection of a second-generation echocardiographic contrast agent. Circulation 1997;96:959-67.

8 Lepper W, Hoffmann R, Kamp O, et al. Assessment of myocardial reperfusion by intravenous myocardial contrast echocardiography and coronary flow reserve after primary percutaneous transluminal coronary angioplasty in patients with acute myocardial infarction. Circulation 2000;101:2368-74
9 Mazur W, Bitar JN, Lechin $M$, et al. Coronary flow reserve may predict myocardial recovery after myocardial infarction in patients with TIMI grade 3 flow. Am Heart J 1998;136:335-44.

10 Gibson CM, Cannon CP, Daley WL, et al. TIMl frame count - a quantitative method of assessing coronary artery flow. Circulation 1996:93:879-88.

11 Iwakura K, Ito H, Takiuchi S, et al. Alternation in the coronary blood flow velocity pattern in patients with no reflow and reperfused acute myocardial infarction. Circulation 1996;94:1269-75.

12 Kawamoto T, Yoshida K, Akasaka T, et al. Can coronary blood flow velocity pattern after primary percutaneous transluminal coronary angioplasty predict recovery of regional left ventricular function in patients with acute myocardial infarction? Circulation 1999;100:33945 .

13 Akasaka T, Yoshida K, Kawamoto T, et al. Relation of phasic coronary flow velocity characteristics with TIMI perfusion grade and myocardial recovery after primary percutaneous transluminal coronary angioplasty and rescue stenting. Circulation 2000;101:2361-7.

14 TIMI Study Group. The thrombolysis in myocardial infarction (TIMI) trial. Phase I findings. N Engl J Med 1985;312:932-6.

15 Schiller NB, Shah PM, Crawford M, et al. Recommendations for quantitation of the left ventricle by two-dimensional echocardiography. American Society of Echocardiography Committee on Standards, subcommittee on quantitation of two-dimensional echocardiograms. J Am Soc Echocardiogr 1989;2:358-67.

16 Kan G, Visser CA, Koolen JJ, et al. Short and long term predictive value of admission wall motion score in acute myocardial infarction. A cross sectional echocardiographic study in 345 patients. Br Heart J 1986:56:422-7.

17 Ito $\mathbf{H}$, Maruyama A, Iwakura K, et al. Clinical implications of the "no reflow" phenomenon. A predictor of complications and left ventricular remodeling in reperfused anterior wall myocardial infarction. Circulation 1996;93:223-8

18 Haager $P$, Christott $P$, Heussen $N$, et al. Prediction of clinical outcome after mechanical revascularization in acute myocardial infarction by markers of myocardial reperfusion. J Am Coll Cardiol 2003:41:532-8.

19 Iwakura K, Ito H, Nishikawa N, et al. Early temporal changes in coronary flow velocity patterns in patients with acute myocardial infarction demonstrating the "no-reflow" phenomenon. Am J Cardiol 1999:84:415-19

20 Wakatsuki T, Nakamura M, Tsunoda T, et al. Coronary flow velocity immediately after primary coronary stenting as a predictor of ventricular wall motion recovery in acute myocardial infarction. J Am Coll Cardiol 2000;35: 1835-41.

21 Lepper W, Sieswerda GT, Franke A, et al. Repeated assessment of coronary flow velocity pattern in patients with first acute myocardial infarction. J Am Coll Cardiol 2002;39:1283-9.

\section{IMAGES IN CARDIOLOGY}

\section{Giant $P$ waves}

A 15 year old girl was referred to our heart failure and transplant clinic with hypertrophic cardiomyopathy. Physical examination revealed signs of right heart failure; echocardiography showed severe concentric hypertrophy of both ventricles in addition to reduced left ventricular systolic function, and moderate dilation of the left atrium and severe dilation of the right atrium. In addition to dilatation, the subcostal view in particular suggested pronounced right atrial hypertrophy. The resting ECG revealed giant $\mathrm{P}$ waves in excess of $1.5 \mathrm{mV}$, amplitude exceeding that of the QRS complex in many leads (right panel). The patient later underwent orthotopic heart transplantation.

T A Simmers

J H Kirkels

t.a.simmers@amc.uva.nl

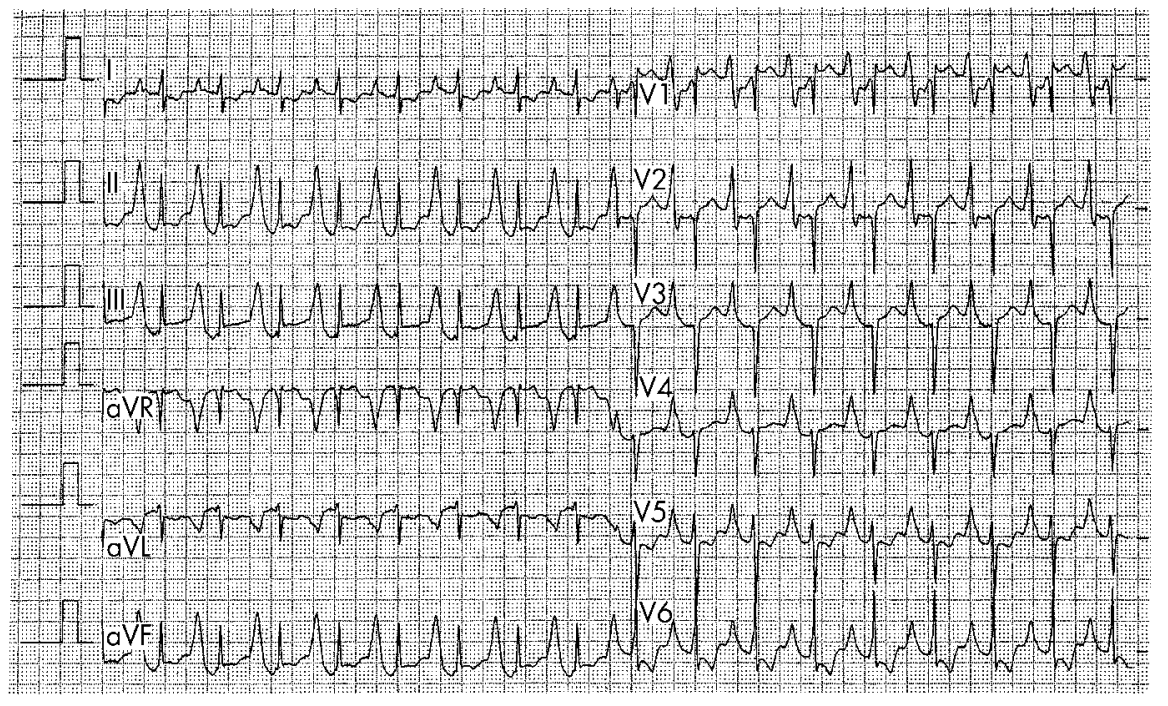

